\title{
Improvement of Handoff Decision in GSM-Railway Using Fuzzy Logic
}

\author{
${ }^{1}$ Deepa Arora, ${ }^{2}$ Sajjan Singh, ${ }^{3}$ Dr. S. V. A. V. Prasad \\ ${ }^{I}$ Student (M. Tech), BRCM Collge of Enginnering and Technology, Bahal, Bhiwani, India \\ 2Assistant Professor, Department of ECE, BRCM College of Engineering \& Technology, Bahal, Bhiwani, India \\ ${ }^{3}$ Professor, Department of ECE, Lingaya's University, Faridabad, India
}

\begin{abstract}
In this paper, we present two algorithms, are based on fuzzy logic, Conventional algorithm and Proposed algorithms. Both handoff algorithms are used average handoff factor to decide when to handoff and when not in case of high speed mobile terminals. Conventional algorithm is of two types, one is Conventional I and other is conventional II. Also Proposed algorithm is of two types, one is proposed I and other is proposed II. The difference among these algorithms is its hysteresis margin. Proposed algorithms are used under the scenario of high speed trains. Proposed algorithm can make handoff efficient at speed of 270-350km/hr. also the probability of unnecessary handoff is reduced.
\end{abstract}

Keywords: Handoff Decision, Fuzzy logic, GSM-R.

\section{Introduction}

GSM was adopted GSM as Europe standard for communication in high speed trains in 1998 [1], named GSM-Railway (GSM-R). it can support efficient communication in railway, especially in high speed trains in china. Its attention to the high speed subscribers is more for getting efficient communication system without any disruption. Due to high speed in passenger special line in china, the handoff decision algorithm could be very efficient, disruption free and more exact than in GSM. Using Conventional algorithm for handoff decision can't provide good performance when mobile station moves with great speed. Because of more "ping pong" effect. It can cause unnecessary handoff. Due to which excessive load occurs on the system. This Unnecessary handoff can occur due to lower signal level but handoff is not required. Until now, many handoff decision algorithms were analyzed for GSM in existing literatures. But, little published work has been devoted to the handoff decision algorithms in GSM-R network.

\subsection{Conventional Algorithms For Handoff Decision:}

Conventional algorithm is based on RSS (received signal strength). And it uses average window for reducing the "ping pong" effect. This effect can be caused by varying RSS. Following shows the diagram of RSS received from current and target BS. Conventional algorithm for handoff decision is of two types one conventional I and other is conventional II. The difference among these two is its hysteresis margin of the RSS.

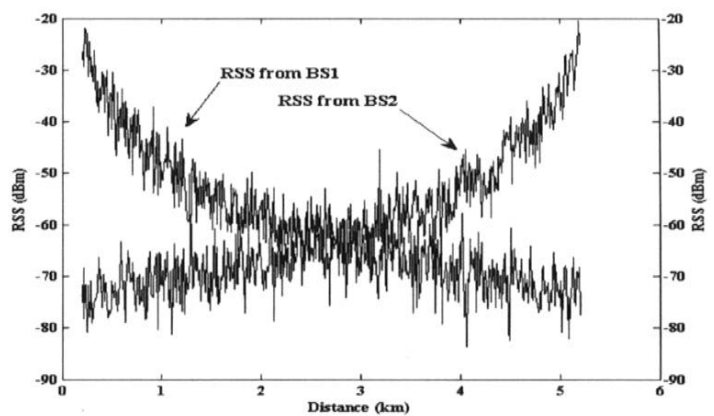

1. RSS received from the current BS and the target BS

Using the hysteresis margin allow a mobile station to make handoff decision only if the value of RSS received of target BS is greater than the RSS received of current BS.

\subsection{Conventional Algorithm I}

RSS received from current $B S-s_{i}(n)$

RSS received from target $B S-s_{j}(n)$

Handoff can happen in conventional algorithm only if the following equation is satisfied.

$\mathrm{Y}_{\mathrm{j}}(\mathrm{n})>\mathrm{Y}_{\mathrm{i}}(\mathrm{n})$ 
$Y_{j}(n)$ and $Y_{i}(n)$ are average $R S S$.

And

$\mathrm{Y}(\mathrm{n})=\frac{1}{N} \sum_{k=n-N+1}^{n} s(k)$

$\mathrm{N}=4$ average window size.

$\mathrm{K}$ is sampling index.

\subsection{Conventional Algorithm Ii} margin.

This algorithm is similar to conventional algorithm II. But only the difference is on its hysteresis

$\mathrm{Y}_{\mathrm{j}}(\mathrm{n})>\mathrm{Y}_{\mathrm{i}}(\mathrm{n})+\mathrm{H}$

is satisfied.

$\mathrm{H}$ is hysteresis margin.

$\mathrm{N}=4$ and $\mathrm{H}=2 \mathrm{dbm}$.

\section{Proposed Algorithms For Handoff Decision}

Proposed algorithm is based on average handoff factor, its value lies between 0 to1. The handoff factor was the output of the proposed FIS (Fuzzy Inference System), which included three inputs: Distance from MT to BS, RSS from BS, Velocity of MT, where RSS is most efficient factor. Distance is easily got from devices; velocity is the key parameter in high speed railway. Using some other factors, such as capacity utilization of BS, signal interferences, etc, could help in finding accurate results, but the result is not accurate because these factors can't be easily and exactly got from the GSM-R. Here, we emphasize dynamic sampling distance in proposed algorithms. In GSM network, most subscribers are moving slowly when using GSM services, so, the sampling distance $1 \mathrm{~m}$ is used for handoff decision in existing literatures. But, in GSM-R network, few subscribers are moving slowly. If sampling distance-lm is still used, the more unnecessary samplings would occur to produce "Ping-Pong" effects. So, dynamic sampling distance we chose was subject to the following equation:

$\mathrm{D}=\mathrm{V} * \mathrm{~T}$

$\mathrm{D}=$ distance from mobile station to BS.

$\mathrm{V}=$ speed of mobile station

$\mathrm{T}=$ sampling time $(480 \mathrm{~ms})$

\section{Proposed algorithm I}

HO_Factor_BS $(\mathrm{n})$ - handoff factor of current BS.

HO_factor_BS $(\mathrm{n})$ - handoff factor of target BS.

These are averaged by average window of size $\mathrm{N}=4$.

In this algorithm handoff occurs when

HO_Factor $(\mathrm{n})>\mathrm{HO} \_$Factor $_{\mathrm{j}}(\mathrm{n})$...

Is satisfied.

HO_Factor $(\mathrm{n})=\frac{1}{N} \sum_{k=n-N+1}^{n} H O_{-}$Factor_BS $(k)$..

Horizontal axis denote distance in $\mathrm{km}$ and vertical axis denote degree of membership

\section{Proposed algorithm II}

It is the enhanced version of proposed algorithm I. In this handoff occurs when

$\mathrm{HO}_{\text {Hactor }}(\mathrm{n})>\mathrm{HO}_{\text {Hactor }}(\mathrm{n})+\mathrm{H}$.

Is satisfied. $\mathrm{H}$ is hysteresis margin.

And $\mathrm{H}=2 \mathrm{dbm}$.

$\mathrm{K}$ is sampling index.

\section{Fuzzification}

We use three inputs, Distance, RSS, and Velocity. And one output Decision of FIS (fuzzy inference system).For improving the performance of handoff in GSM-R. We designed four fuzzy sets- decision set, distance set, RSS set and velocity set. Using FIS we could get handoff factor without averaging from Decision set. Where " 0 " means no handoff and " 1 " means handoff occurs. 


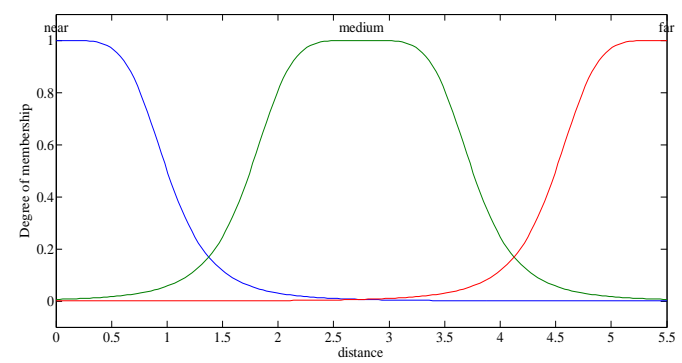

2 Membership function of distance

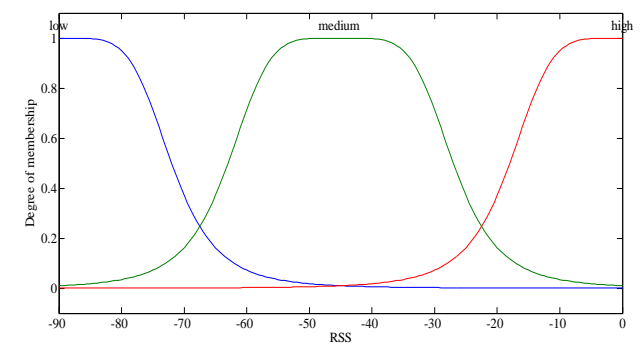

3 Membership functions of Rss

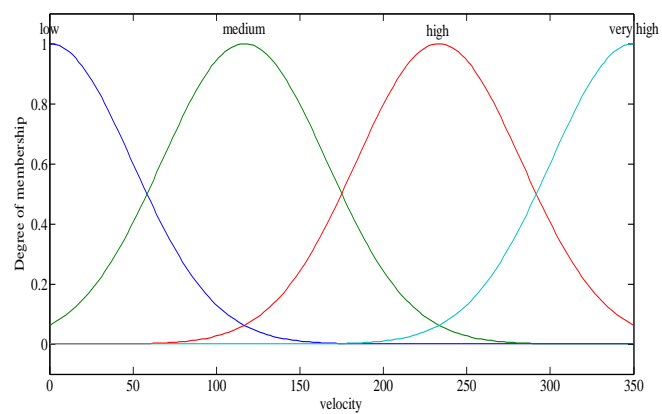

4 Membership functions of velocity

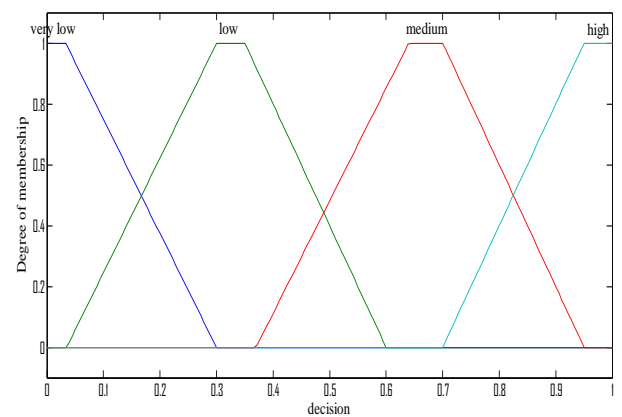

5 Membership Function of Decision

\subsection{Fuzzy Inference Engine}

The fuzzy inference engine is a group of rules developed using expert knowledge. We have designed the knowledge-based rules that connect the inputs and the outputs based on a careful understanding of the philosophy behind the handover behaviors in GSM-R. The fuzzy inference system is designed based on following rules, which are presented in Figure. In order to demonstrate the designed fuzzy inference system, one rule is used to show how the inference engine works and the outputs of each rule are combined to generate the fuzzy decision.

R1: IF Distance is near and RSS is high and velocity is very high THEN NO_Handoff.

R2: IF Distance is near and RSS is high and velocity is high THEN NO_Handoff.

R3: IF Distance is near and RSS is high and velocity is medium THEN NO_Handoff.

R4: IF Distance is near and RSS is high and velocity is low THEN NO_Handoff.

R5: IF Distance is near and RSS is medium and velocity is very high THEN WAIT.

R6: IF Distance is near and RSS is medium and velocity is high THEN WAIT.

R7: IF Distance is near and RSS is medium and velocity is medium THEN No_Handoff. 
R8: IF Distance is near and RSS is medium and velocity is low THEN No_Handoff. R9: IF Distance is near and RSS is low and velocity is very high THEN Be_Careful. R10: IF Distance is near and RSS is low and velocity is high THEN WAIT.

R11: IF Distance is near and RSS is low and velocity is medium THEN No_Handoff. R12: IF Distance is near and RSS is low and velocity is low THEN No_Handoff.

R13: IF Distance is medium and RSS is high and velocity is very high THEN Handoff.

R14: IF Distance is medium and RSS is high and velocity is high THEN Be_Careful.

R15: IF Distance is medium and RSS is high and velocity is medium THEN WAIT.

R16: IF Distance is medium and RSS is high and velocity is low THEN No_Handoff.

R17: IF Distance is medium and RSS is medium and velocity is very high THEN Handoff.

R18: IF Distance is medium and RSS is medium and velocity is high THEN Handoff.

R19: IF Distance is medium and RSS is medium and velocity is medium THEN Be_Careful.

R20: IF Distance is medium and RSS is medium and velocity is low THEN WAIT.

R21: IF Distance is medium and RSS is low and velocity is very high THEN Handoff.

R22: IF Distance is medium and RSS is low and velocity is high THEN Handoff.

R23: IF Distance is medium and RSS is low and velocity is medium THEN Be_Careful.

R24: IF Distance is medium and RSS is low and velocity is low THEN Be_Careful.

R25: IF Distance is far and RSS is high and velocity is very high THEN Be_Careful.

R26: IF Distance is far and RSS is high and velocity is high THEN Be_Careful

R27: IF Distance is far and RSS is high and velocity is medium THEN WAIT.

R28: IF Distance is far and RSS is high and velocity is low THEN WAIT.

R29: IF Distance is far and RSS is medium and velocity is very high THEN Handoff.

R30: IF Distance is far and RSS is medium and velocity is high THEN Handoff.

R31: IF Distance is far and RSS is medium and velocity is medium THEN Be_careful.

R32: IF Distance is far and RSS is medium and velocity is low THEN WAIT.

R33: IF Distance is far and RSS is low and velocity is very high THEN Handoff.

R34: IF Distance is far and RSS is low and velocity is high THEN Handoff.

R35: IF Distance is far and RSS is low and velocity is medium THEN Handoff.

R36: IF Distance is far and RSS is low and velocity is medium THEN

\subsection{Block Diagram Of Fuzzy Algorithm}

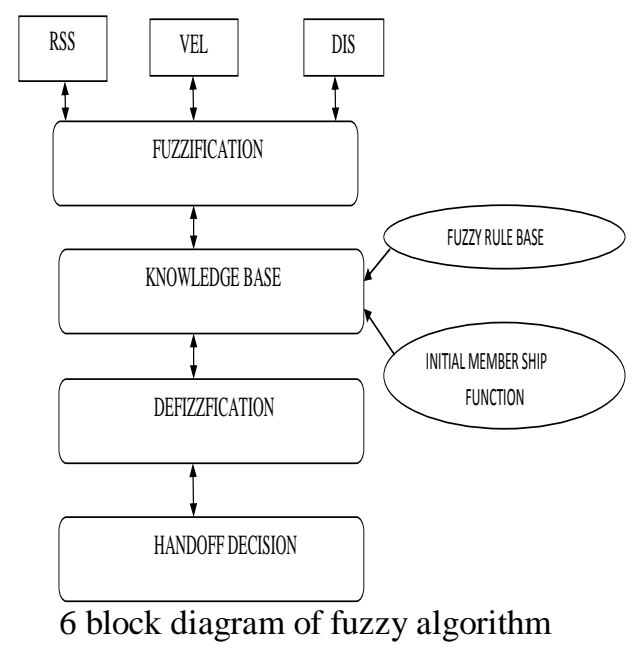

This is the block diagram of the fuzzy algorithm, where RSS, Velocity and Distance are the input parameters. Which are firstly fuzzified these inputs are applied to the knowledge base which is formed by the fuzzy rule base. And the initial membership functions of the inputs are applied to the knowledge base rule and then this value is deffuzified means it is converted to crisp set. And then we will the handoff status shown in the following block diagram.

\subsection{COMPARISON BETWEEN CONVENTIONAL, PROPOSED I AND PROPOSED II ALGORITHM}

Comparison between conventional, Proposed I and Proposed II algorithm for handoff decision is done here by using fuzzy logic. This coding is based on MATLAB simulation. 
By using this coding for comparison among these three handoff decision algorithm, we can decide which one algorithm is best suited for handoff decision. We can also decide in which algorithm number of errored handoff is small.

\section{Simulations And Results}

These two proposed fuzzy logic based handoff decision algorithms are evaluated in the one of many deployments in Passenger Special Line in China-Two BSs are set in straight line along the trajectory of high speed railway (see Fig. 6). And, given that the handoff execution was successful when the handoff decision occurred.

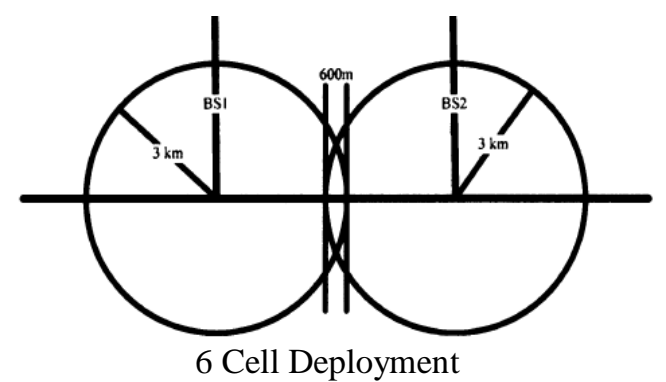

In the simulation, we assumed that the MT started from the place near to BS1 to BS2 and communicated successfully with the BS1 at the beginning.

The detailed information of simulation parameters is given in the table below. We simulated two conventional and two proposed algorithms in the specified scenario.

\section{Graphical response of conventional}

\section{algorithm}

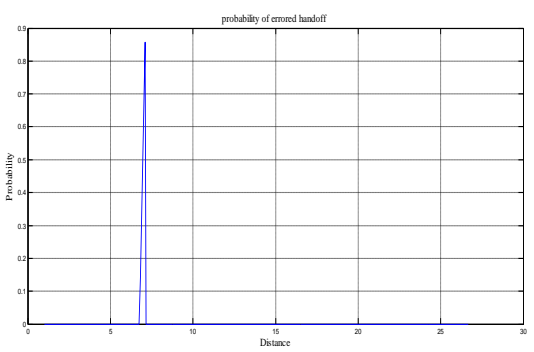

Fig.7 Probability of unnecessary handoff

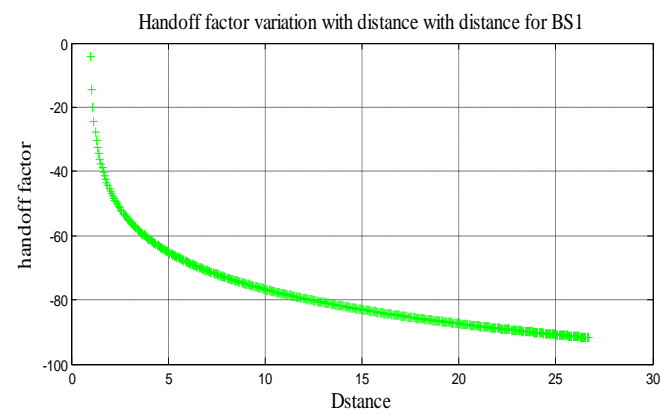

Fig. 8 Handoff factor variation with distance

\section{Graphical results of proposed I algorithm}

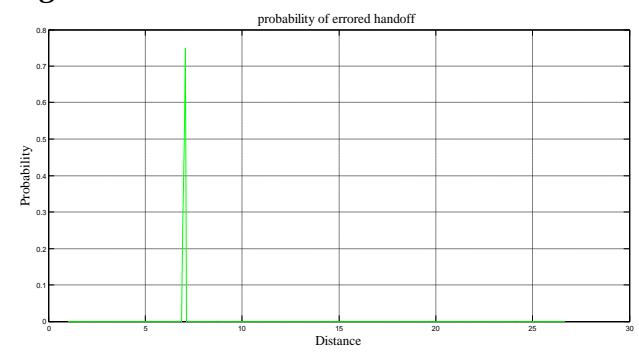

Fig. 9. Probability of errored handoff 


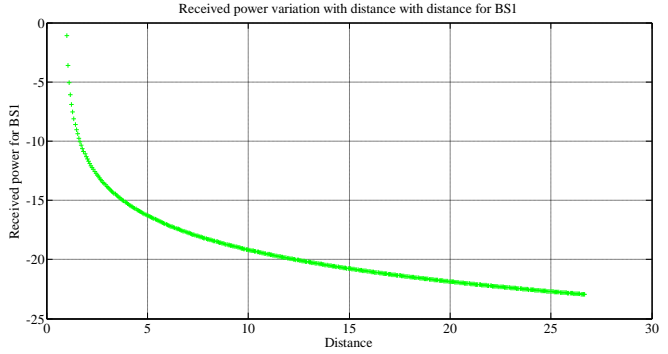

Fig. 10 Received power variation with distance

\section{Graphical results of Proposed II algorithm}

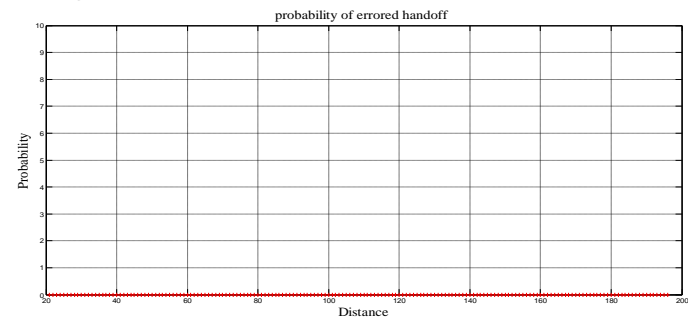

11 Probability of errored handoff

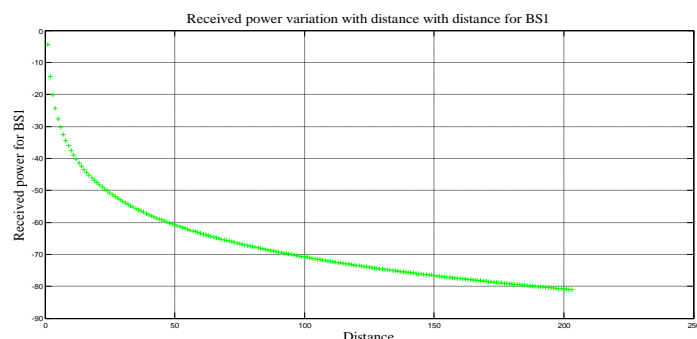

12 Received power variation with distnce

9.4 Graphical results of comparison between Conventional, Proposed I and Proposed II algorithm

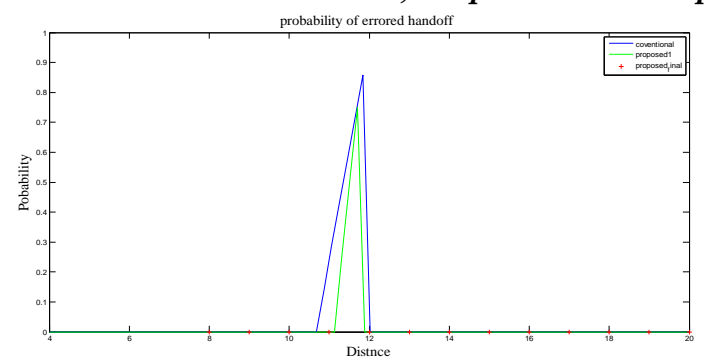

13 Probability of errored handoff

\section{Conclusion}

As from the comparison, we can see that proposed-2 algorithm for handoff decision is best among these three handoff decision algorithm. Because the probability of errored or unnecessary handoff in minimum or negligible but the rest of two handoff deciding algorithm has higher probability of errored handoff. Proposed-2 algorithm is best suited for deciding handoff decisions. Also the received power for BS1 is more in case of proposed-2 handoff decision algorithm then other two algorithms.

\section{References}

[1] T.S. Rappaport, wireless communication, Principle and practice. Upper Saddel River, NJ: Prentice Hall, 1996.

[2] V.K.Garg and J.E Wilkes, Wireless and Personal Communications System: Prentice Hall inc., 1996.

[3] C.Briso, C. Cortes, F.J. Arques, J.I. Alonso "Requirement of GSM Technology for the control of high speed trains". The 13th IEEE international symposium on personal, indoor and Mobile radio communication, Volume 2(2002).

[4] G. Edwards, R. Sankar. "Handoff Using Fuzzy Logic",IEEGlobal Telecommunications Conference, volume 1(1995).

[5] H.L. Nan, X. Wang, Z.D. Zhong. "Study on algorithm of cell coverage Radius in GSM-R" in Journal of The China Railway Society, Volume 27.(2005). 